\title{
Developmental Expression of Penile Reflexes and Copulatory Behavior in Male Rats
}

\author{
Toru R. SAITO1), Misao TERADA1), Naoki MORITANI1), \\ Haruo HASHIMOTO'1), Toshihiro OIKAWA2), and Satoshi SOETA2)
}

\begin{abstract}
1)Behavioral Neuroscience Laboratory, Department of Laboratory Animal Sciences, and 2)Department of Veterinary Anatomy, Nippon Veterinary and Animal Science University, Musashino, Tokyo 180-8602, Japan
\end{abstract}

\begin{abstract}
First, pubertal development of the penile reflexes, e.g., erections, cups and flips in Wistar-Imamichi male rats was investigated following sheath retraction. Second, the penile reflexes and copulatory behavior in the above males were compared between 10 and 44 weeks of age. The penile reflexes in Wistar-Imamichi rats began to appear from day 26, and all males displayed full components of the reflexes on day 47. The occurrence rates and mean numbers of erections, cups and flips in aged adults were significantly low, compared with the young adults. Also, in the observation of copulatory behavior the occurrence rate of ejaculations, and mean numbers of intromissions and ejaculations in aged adults were significantly lower than that in young adults. These results of the present study may suggest that the decrease of copulatory behavior in male rats with age results from the dysfunction of the penile reflexes with age.
\end{abstract}

Key words: Aging, male rats, penile reflexes

It is well known that age-dependent changes are prominent in reproductive systems. Normal men experience a gradual decrease not only in sexual activity with age, but also in libido or interest in sexual activity [6]. In spite of the fact that occasional cases of fertility persisting in men in their 90's have been reported, fertility rates decline in men during the normal aging process [2]. Similarly, in normal nonhuman primates and in other mammals such as rats, various measures of male sexual activity decline with aging. Rates of contact with females, mounting, intromission and ejaculation all decrease in normal old animals [7, 10].
Saito et al. [15] and Hokao et al. [4, 5] demonstrated that conception rates after copulation in aged adult rats decreased compared with young adults.

Sachs [14] reported that similar to other mammalian species, male rats display penile erection in several contexts, reflexive erections can be evoked from supine, conscious rats by the tonic stimulation of the retracted penile sheath at the base of the glans penis [9]. The questions addressed here were whether the potency of penile reflex, like the sexual activity, in male rats would decrease with age, and whether a relationship between the potencies of penile reflexes and copulatory behav-

(Received 18 July 2002 / Accepted 26 November 2002)

Address corresponding: T.R. Saito, Department of Laboratory Animal Sciences, Nippon Veterinary and Animal Science University, 1-7-1 Kyonan-cho, Musashino-shi, Tokyo 180-8602, Japan 
ior could be seen with age.

The present study was performed as follows. The pubertal development of the penile reflexes in male rats was investigated. After the above males displayed penile reflexes, two items, the penile reflexes and copulatory behavior, were observed with age.

Subjects: Pregnant rats of Wistar-Imamichi strain were obtained from Imamichi Institute for Reproduction (Tsuchiura, Ibaraki) about ten days before parturition. The animals were housed in individual polycarbonate cages $(22 \mathrm{~W} \times 38 \mathrm{D} \times 20 \mathrm{H} \mathrm{cm})$ with wood shavings as bedding and kept at $24 \pm 2{ }^{\circ} \mathrm{C}$ in a light-controlled room (14 h light, $10 \mathrm{~h}$ darkness). Food (Oriental MF, Oriental Yeast Co., Ltd., Tokyo) and tap water were available ad libitum. The day of parturition was designated as day 0 of lactation and number of pups per litter was adjusted to eight within 2 days of birth. Littermates were weaned at 21 days of age and randomly assigned to the testing for penile reflexes. Upon weaning, males were earpunched for identification and housed, 4-5 per cage, in hanging wire mesh cages $(31 \mathrm{~W} \times 44 \mathrm{D} \times 23 \mathrm{H} \mathrm{cm})$.

Penile reflex test: The test of penile reflexes began at 23 days of age and continued every 3 days until males displayed all of erections, cups and flips between 16:00 and 17:00. Penile reflexes were tested after a method developed by Hart [3] and modified by Sachs and Garinello [12]. Briefly, each unanesthetized male was placed on his back with the anterior portion of his body loosely enclosed with a belt around his midsection. The penile sheath was retracted to the extent possible and held in position with tape. The maintenance in that position produces light pressure at the base of the sheath which provokes phasic episodes of penile responses without any further stimulation (Fig. 1). Males were tested for 15 min following the initiation of reflexes or $30 \mathrm{~min}$ in the event of no reflexes occurring. The occurrence rates and frequencies of erections, cups and flips were recorded on an event recorder as they occurred. Erections are extensions and / or distensions of the penis. Cups are intense erections during which the glans of the penis flares so that it is more trumpet-shaped than tubular. Flips are a dorsal flexion of the penis. For comparison of penile reflexes with aging, the number of each type of reflex was meas-

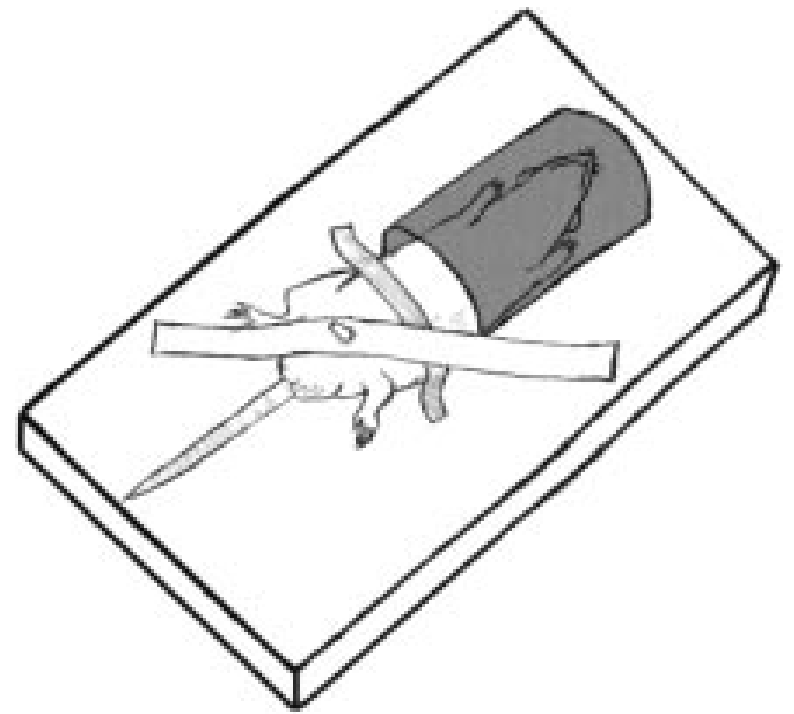

Fig. 1. Testing situation for evocation of penile responses ex copula.

ured and recorded on reaching the ages of 10 and 44 weeks.

Copulatory behavior test: After the penile reflex test, the above males were subjected to copulatory behavior testing at 10 and 44 weeks of age. Sexually receptive females aged 8 weeks, whose vaginal smears had proestrous features after consecutive repetitions of 2 cycles of the 4-day estrous cycle, were selected. After a male rat was placed in the semi-circular observation cage (radius 40 , height $50 \mathrm{~cm}$ ) faced with Plexiglass under low-level red-light illumination for a few minutes, a sexually receptive female was introduced to the cage. Tests lasted $2 \mathrm{~h}$ from the introduction of the female. Behavioral testing was conducted between 19:30 and 21:30. The behavior categories scored included the following [15]. Mount frequency: number of mounts without intromission during $2 \mathrm{~h}$. Intromission frequency: number of mounts with intromission during $2 \mathrm{~h}$. Ejaculation frequency: number of ejaculations during $2 \mathrm{~h}$.

Statistics: For comparison of incidence of penile reflexes and copulatory behaviors between groups, Fisher's exact probability test [1] was used. Behavioral frequencies were analyzed using Student's paired $t$-test to compare the mean values. 


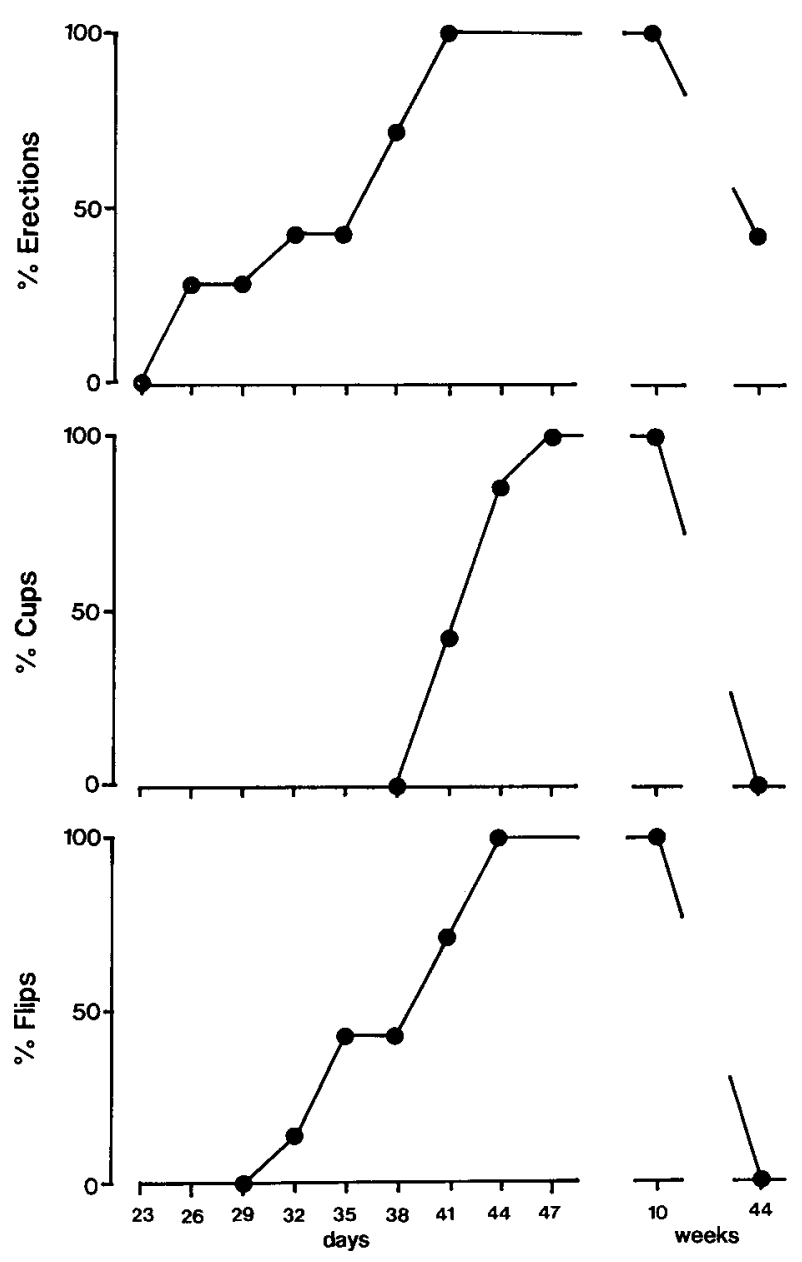

Fig. 2. Cumulative frequency curves for age of occurrence of first erection, cup and flip in male rats.

Pubertal development of the penile reflexes: The cumulative frequency curves for the development of penile reflexes in seven male rats are shown in Fig. 2. Erections were first seen on day 26. All of 7 male rats displayed erections on day 41. Cups were observed after 41 days and all 7 males showed cups by day 47 . Flips occurred on day 32 and all displayed flips by day 44. The penile reflexes in Wistar-Imamichi rats began to appear from day 26, and all males displayed full components of the reflexes on day 47 .

Penile reflexes with age: The comparison of occurrence rates and numbers of erections, cups and flips in male rats aged 10 and 44 weeks are shown in Fig. 3. All of 7 young adult males displayed erections, cups and flips. Mean numbers of erections, cups and flips were $16.1 \pm 4.0$ (s.e.m.), $8.9 \pm 2.7$ and $4.0 \pm 2.4$, respectively. In contrast, of 7 aged adults three showed erections, but no cups and flips. Mean number of erections was $0.4 \pm 0.2$. The occurrence rates and mean numbers of erections, cups and flips in aged adults were significantly low $(\mathrm{p}<0.05-0.01)$, compared with these in young adults.

Copulatory behavior with age: The comparison of occurrence rates and numbers of mounts, intromissions and ejaculations in male rats aged 10 and 44 weeks are shown in Fig. 4. All 7 young adults displayed mounts, intromissions and ejaculations. Mean numbers of mounts, intromissions and ejaculations were $33.8 \pm 3.7$ (s.e.m.), $42.4 \pm 3.0$ and $6.8 \pm 0.4$, respectively. Contrary to the young adults, in aged adults mean numbers (and occurrence rates) of mounts, intromissions and ejaculations were $60.2 \pm 8.4$ (7/7), $8.0 \pm 4.5(6 / 7)$ and $1.6 \pm 1.0(3 / 7)$, respectively. There were significant differences in the occurrence rates of ejaculation $(\mathrm{p}<0.05)$, and mean numbers of intromissions and ejaculations $(\mathrm{p}<0.01)$ between young and aged adults.

First, the pattern of pubertal development of the penile reflexes in male rats was observed. The development of erections was markedly faster than cups which developed later. The difference of development in erections, cups and flips seems to result from the mechanical bases of penile actions. Sachs and Meisel [11] indicated that the penile reflexes in Long-Evans rats developed preceding the time of maximum testosterone levels at 50 days of age. Our data of the present study also showed that the penile reflexes were complete by day 47 , before a peak level of testosterone $(3.1 \pm 0.8 \mathrm{ng} / \mathrm{ml})$ on day 51 [16]. The pubertal development of penile reflexes in intact and spinally transected male rats was investigated by Meise and Sachs [8]. They found that spinal transection advanced the ages of onset of penile erections and flips about 10 days relative to control males. These results suggest that the pubertal development of penile actions is dependent upon the spinal control rather than blood testosterone levels.

Second, we observed the potencies of penile reflexes and copulatory behavior with age using the same rats. Previous data regarding patterns of copulatory behavior with age, but not penile reflexes with age have been reported in a few species [2, 4-7, 10, 15]. Hokao et al. 

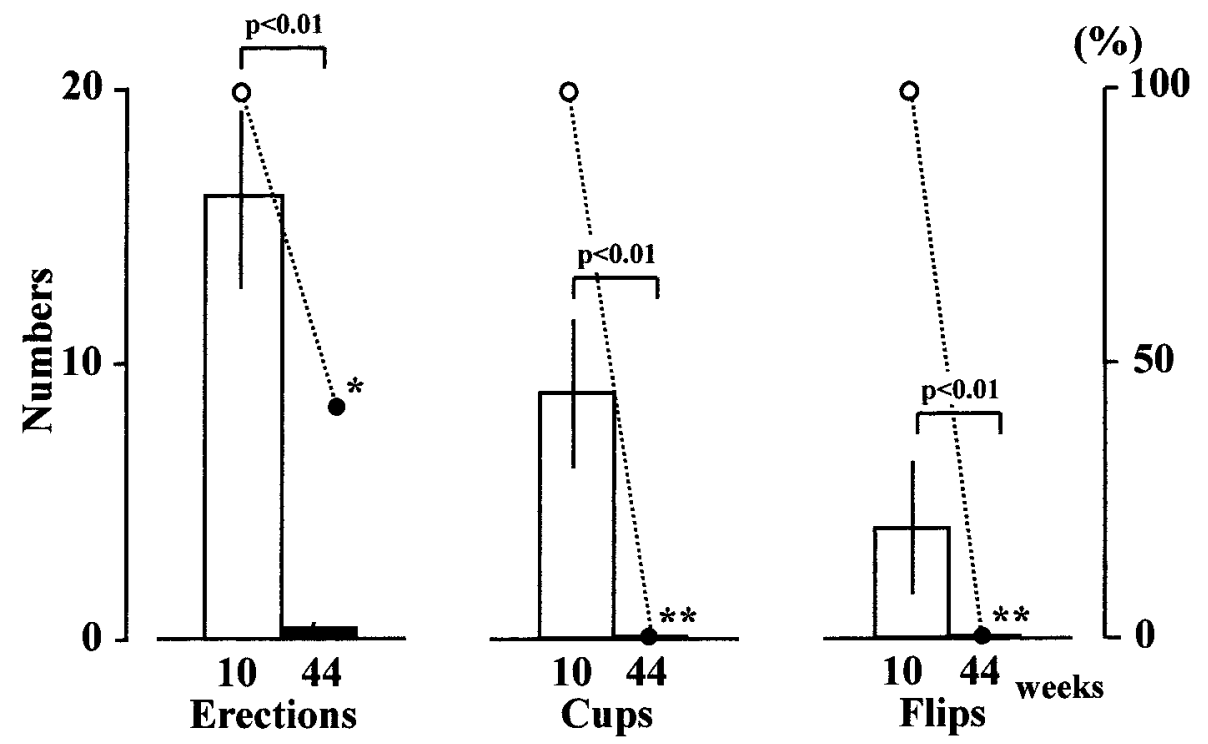

Fig. 3. Occurrence rates (circles) and frequencies (bars) of penile reflexes between young and aged adult rats. Data are Mean (bars) \pm s.e.m. (lines), *: $\mathrm{p}<0.05, * *: \mathrm{p}<0.01$.
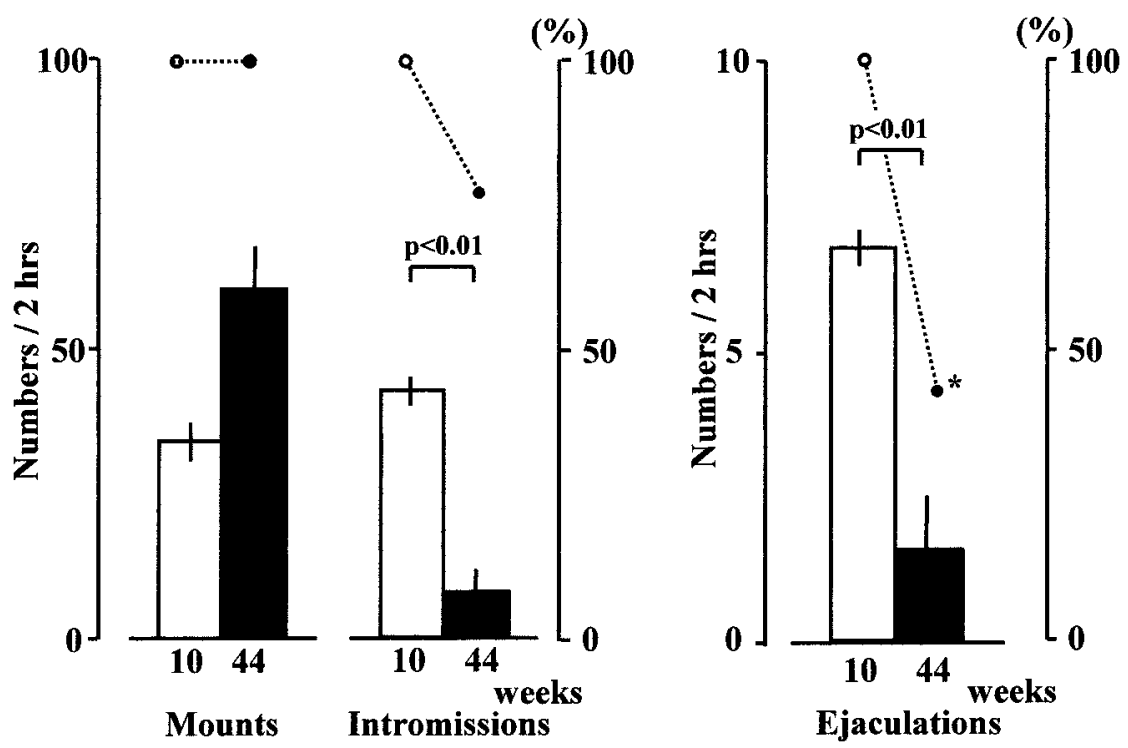

Fig. 4. Occurrence rates (circles) and frequencies (bars) of copulatory behavior between young and aged adult rats. Data are Mean (bars) \pm s.e.m. (lines), *: $p<0.05$.

$[4,5]$ investigated copulatory behavior in WistarImamichi male rats at 10, 27, 44, 67 and 104 weeks of age. The results indicated that the items of copulatory behavior affected in order of age were ejaculation, intromission and mount. Upon reaching 44 and 67 weeks of age, the occurrence rates of ejaculations, intromissions and mounts were approximately 40,80 and $100 \%$, respectively. Eventually the ejaculation and intromission disappeared, but mount lasted with a $40 \%$ occurrence rate at 104 weeks of age. Similarly, our data showed a significant decrease of ejaculations and intromissions, but not mounts at 44 weeks of age, compared with 10 weeks of age. These data indicate no age-related change in rat mounting. On the other hand, 
the potency of penile reflexes with age likewise decreased. At 44 weeks of age the cup and flip, but not erection disappeared during our observation time, suggesting that the latencies of cup and flip were longer than these at 10 weeks of age. Given a longer observation time, the cup and flip might appear. It is considered that the perineal muscles related to penile reflexes in male rats and mice are as follows; $\mathrm{M}$. ischiocavernosus, M. bulbocavernosus and M. levator ani. From these muscle excision studies, the role of striated penile muscles in penile reflexes was clarified by Sachs [13]. $\mathrm{He}$ found that the ischiocavernosus and bulbocavernosus muscles activities predominated during erections, the ischiocavernosus muscle activity predominated during erections and flips, and the bulbocavernosus muscle activity predominated during cups. These findings suggest that age-related differences of erections, cups and flips in Wistar-Imamichi rats might be explained by age-related functional changes of striated penile muscles in penile reflexes. Histological studies with age will be necessary in order to clarify these points in penile reflexes.

In conclusion, the present study is the first to clearly establish the pattern of penile reflexes with age and the relationship between penile reflexes and copulatory behavior with age in male rats.

\section{Acknowledgments}

The authors thank Dr. Y. Kondo, Department of Physiology I, Nippon Medical School, for technical assistance. This study was supported in part by grants-in-aid for Scientific Research from the Ministry of Education, Science and Culture of Japan (No. 09460148) to TRS. A preliminary report of this re- search was presented at the 15th Annual Meeting of the Japan Society of Andrology, Kurashiki, July 1996.

\section{References}

1. Gad, S.C. and Weil C.S. 1982. pp. 291-292. In: Principles and methods of toxicology (Hayes, A.W., ed), Raven Press, New York.

2. Harman, S.M. 1978. pp. 29-58. In: Aging reproductive system. (Schneider, E.L.,ed.), Raven Press, New York.

3. Hart, B.L. 1968. J. Comp. Physiol. Psychol. 65: 453-460.

4. Hokao, R., Ito, T., Noguchi, J., Wakafuji, Y., Agematsu, Y., Saito, T.R., Takahashi, K.W., and Imamichi, T. 1992. Exp. Anim. 41: 259-268.

5. Hokao, R., Saito, T.R., Wakafuji, Y., Takahashi, K.W., and Imamichi, T. 1993. Exp. Anim. 42: 75-82.

6. Kinsey, A.C., Pomeroy, W.B., and Martin, C.E. 1948. pp. 1-789. In: Sexual behavior in the human male (Kinsey, A.C., Pomeroy, W.B., and Martin, C.E., eds),. Sanders, Philadelphia.

7. Larsson, K. and Essberg, L. 1962. Gerontologia 6: 133134.

8. Meisel, R.L. and Sachs, B.D. 1980. Physiol. Behav. 24: 289-292.

9. Meisel, R.L. and Sachs, B.D. 1994. 3-105. In: The Physiology of Reproduction (Knobil, E. and Neill, J.D., eds.), Raven, New York.

10. Phoenix, C.G. and Chambers, K.C. 1982. pp. 95-104. In: Advanced views in primate biology (Chiarelli, A.B. and Corrucini, R.S., eds.), Springer, Berlin.

11. Sachs, B.D. and Garinello, L.D. 1978. J. Comp. Physiol. Psychol. 92: 759-767.

12. Sachs, B.D. and Meisel, R.L. 1979. Psychoneuroendocrinology 4: 287-296.

13. Sachs, B.D. 1982. J. Reprod. Fertil. 66: 433-443.

14. Sachs, B.D. 1995. Neurosci. Biobehav. 19: 211-224.

15. Saito, T.R., Hokao, R., Aoki, S., Chiba, N., Terada, M., Saito, M., Dohbutsu, A., Amao, H., Wakafuji, Y., Sugiyama, M., and Takahashi, K.W. 1991. Exp. Anim. 40: 337-341.

16. Saito, T.R., Moritani, N., and Katsuyama, M. 2002. Scand. J. Lab. Anim. Sci. 29: 142-148. 